\title{
Geometry of the Solutions of Localized Induction Equation in the Pseudo-Galilean Space
}

\author{
Muhittin Evren Aydin, ${ }^{1}$ Adela Mihai, ${ }^{2}$ Alper Osman Ogrenmis, ${ }^{1}$ and Mahmut Ergut ${ }^{3}$ \\ ${ }^{1}$ Department of Mathematics, Faculty of Science, Firat University, 23200 Elazig, Turkey \\ ${ }^{2}$ Department of Mathematics and Computer Science, Technical University of Civil Engineering Bucharest, 020396 Bucharest, Romania \\ ${ }^{3}$ Department of Mathematics, Faculty of Science and Letters, Namik Kemal University, 59030 Tekirdağ, Turkey
}

Correspondence should be addressed to Adela Mihai; adela_mihai@fmi.unibuc.ro

Received 26 January 2015; Accepted 21 May 2015

Academic Editor: Soheil Salahshour

Copyright (C) 2015 Muhittin Evren Aydin et al. This is an open access article distributed under the Creative Commons Attribution License, which permits unrestricted use, distribution, and reproduction in any medium, provided the original work is properly cited.

We study the surfaces corresponding to solutions of the localized induction equation in the pseudo-Galilean space $\mathbb{G}_{3}^{1}$. We classify such surfaces with null curvature and characterize some special curves on these surfaces in $\mathbb{G}_{3}^{1}$.

\section{Introduction}

The localized induction equation (LIE), also called the vortex filament equation or the Betchov-Da Rios equation, is an idealized model of the evolution of the centerline of a thin vortex tube in a three-dimensional inviscid incompressible fluid. The connection of LIE with the theory of solitons was discovered by Hasimoto proving that the solutions of LIE are related to solutions of the cubic nonlinear Schrödinger equation, which is well known to be an equation with soliton solutions. For details, see [1-5].

The soliton surface associated with the nonlinear Schrödinger equation is called a Hasimoto surface. The geometric properties of such surfaces are investigated in $[4,6,7]$. By motivating the fact that the study of the Hasimoto surfaces can be interesting in the pseudo-Galilean space which is one of the Cayley-Klein spaces, we are mainly interested in the geometric properties of these surfaces in the pseudo-Galilean space.

Let $(M, g)$ be a 3-dimensional Riemannian manifold and $\nabla$ the Levi-Civita connection with respect to $g$. Note that the cross product of two vector fields $X, Y$ on $M$ may be defined as

$$
g(X \times Y, Z)=d v_{g}(X, Y, Z)
$$

where $d v_{g}$ is the volume form of the manifold and $X, Y, Z \in$ $\Gamma(T M)$. Denote by $\mathbf{r}=\mathbf{r}(s, t)$ the vortex filament; then LIE or the Betchov-Da Rios filament equation is

$$
\mathbf{r}_{t}=\mathbf{r}_{s} \times \nabla_{\mathbf{r}_{s}} \mathbf{r}_{s} .
$$

On the other hand, the Galilean space which can be defined in three-dimensional projective space $P_{3}(\mathbb{R})$ is the space of Galilean Relativity. The geometries of Galilean space and pseudo-Galilean space have similarities but, of course, are different. For study of surfaces in the pseudo-Galilean space, we refer to Šipuš and Divjak's series of works [8-13].

In classical geometry of surfaces, it makes sense to classify the surfaces having null curvature. In particular, a surface is said to be developable if it has null Gaussian curvature. In this case the surface can be flattened onto a plane without distortion. We remark that cylinders and cones are examples of developable surfaces, but the spheres are not under any metric.

There exist significant applications of the results obtained on the surfaces of null curvature in different fields, for example, in microeconomics. When the graphs of production functions in microeconomics have null Gaussian curvature, one can realize a "good" analysis of isoquants by projections, without losing essential information about their geometry 
(see $[14,15])$. By targeting that, we study the Hasimoto surfaces in the pseudo-Galilean space $\mathbb{G}_{3}^{1}$.

In this paper, we classify the Hasimoto surfaces having null curvature in $\mathbb{G}_{3}^{1}$. Then we analyze the parameter curves on such surfaces from the point of view to be the geodesics, asymptotic lines, and principal curves in $\mathbb{G}_{3}^{l}$.

\section{Preliminaries}

The pseudo-Galilean space $\mathbb{G}_{3}^{1}$ is one of the Cayley-Klein spaces with absolute figure that consists of the ordered triple $\{\omega, f, I\}$, where $\omega$ is the absolute plane in the threedimensional real projective space $P_{3}(\mathbb{R}), f$ is the absolute line in $\omega$, and $I$ is the fixed hyperbolic involution of points of $f$ $[8,9,13]$.

Homogenous coordinates of $\mathbb{G}_{3}^{1}$ can be given in the following way: The absolute plane $\omega$ is given by $x_{0}=0$, the absolute line $f$ by $x_{0}=x_{1}=0$, and the hyperbolic involution by $\left(0: 0: x_{2}: x_{3}\right) \mapsto\left(0: 0: x_{3}: x_{2}\right)$, which is equivalent to the requirement for the conic $x_{2}^{2}-x_{3}^{2}=0$ to be the absolute conic. The metric connections in $\mathbb{G}_{3}^{1}$ are introduced with respect to the absolute figure. According to the affine coordinates given by $\left(x_{0}: x_{1}: x_{2}: x_{3}\right)=(1: x:$ $y: z)$, the distance between the points $\mathbf{X}=\left(x_{1}, x_{2}, x_{3}\right)$ and $\mathbf{Y}=\left(y_{1}, y_{2}, y_{3}\right)$ is defined by (see $\left.[16]\right)$

$$
d(\mathbf{X}, \mathbf{Y})= \begin{cases}\left|y_{1}-x_{1}\right|, & \text { if } x_{1} \neq y_{1} \\ \sqrt{\left|\left(y_{2}-x_{2}\right)^{2}-\left(y_{3}-x_{3}\right)^{2}\right|,} & \text { if } x_{1}=y_{1}\end{cases}
$$

The pseudo-Galilean scalar product of the vectors $\mathbf{X}=$ $\left(x_{1}, x_{2}, x_{3}\right)$ and $\mathbf{Y}=\left(y_{1}, y_{2}, y_{3}\right)$ is defined by

$$
\mathbf{X} \cdot \mathbf{Y}= \begin{cases}x_{1} y_{1}, & \text { if } x_{1} \neq 0 \text { or } y_{1} \neq 0 \\ x_{2} y_{2}-x_{3} y_{3}, & \text { if } x_{1}=0, \quad y_{1}=0\end{cases}
$$

In this sense, the pseudo-Galilean norm of a vector $\mathbf{X}$ is $\|\mathbf{X}\|=$ $\sqrt{|\mathbf{X} \cdot \mathbf{X}|}$. A vector $\mathbf{X}=\left(x_{1}, x_{2}, x_{3}\right)$ is called isotropic if $x_{1}=$ 0 ; otherwise it is called nonisotropic. All unit nonisotropic vectors are of the form $\left(1, x_{2}, x_{3}\right)$. The isotropic vector $\mathbf{X}=$ $\left(0, x_{2}, x_{3}\right)$ is called spacelike, timelike, and lightlike if $x_{2}^{2}-x_{3}^{2}>$ $0, x_{2}^{2}-x_{3}^{2}<0$, and $x_{2}= \pm x_{3}$, respectively. The pseudoGalilean cross product of $\mathbf{X}$ and Yon $\mathbb{G}_{3}^{1}$ is given by

$$
\mathbf{X} \times \mathbf{Y}=\left|\begin{array}{ccc}
0 & -\mathbf{e}_{2} & \mathbf{e}_{3} \\
x_{1} & x_{2} & x_{3} \\
y_{1} & y_{2} & y_{3}
\end{array}\right|
$$

where $\mathbf{e}_{i}=\left(\delta_{i 1}, \delta_{i 2}, \delta_{i 3}\right), 1<i \leq 3$.

Let $\gamma$ be an admissible curve given by $\gamma(s)=$ $\left(\gamma_{1}(s), \gamma_{2}(s), \gamma_{3}(s)\right)$ in $\mathbb{G}_{3}^{1}$; that is, it has no isotropic tangent vectors for $\forall s$. Note that an admissible curve has nonzero curvature. Then its curvature and torsion functions are, respectively, defined by

$$
\begin{aligned}
& \kappa(s)=\frac{\sqrt{\left|\left(\ddot{\gamma}_{2}(s)\right)^{2}-\left(\ddot{\gamma}_{3}(s)\right)^{2}\right|}}{\left(\dot{\gamma}_{1}(s)\right)^{2}}, \\
& \tau(s)=\frac{\ddot{\gamma}_{2}(s) \ddot{\gamma}_{3}(s)-\dddot{\gamma}_{2}(s) \ddot{\gamma}_{3}(s)}{\left|\dot{\gamma}_{1}(s)\right|^{5} \kappa^{2}(s)},
\end{aligned}
$$

where $\dot{\gamma}_{i}(s)=d \gamma_{i} / d s$ and so forth, $1 \leq i \leq 3$. If $\gamma$ is an admissible curve parameterized by the arc length $s$, it is of the form

$$
\gamma(s)=\left(s, \gamma_{2}(s), \gamma_{3}(s)\right) .
$$

The associated Frenet frame field of $\gamma$ is the trihedron $\{\mathbf{T}, \mathbf{N}, \mathbf{B}\}$ such that

$$
\begin{aligned}
& \mathbf{T}(s)=\dot{\gamma}(s)=\left(1, \dot{\gamma}_{2}(s), \dot{\gamma}_{3}(s)\right), \\
& \mathbf{N}(s)=\frac{1}{\kappa(s)} \ddot{\gamma}(s)=\frac{1}{\kappa(s)}\left(0, \ddot{\gamma}_{2}(s), \ddot{\gamma}_{3}(s)\right), \\
& \mathbf{B}(s)=\frac{1}{\kappa(s)}\left(0, \epsilon \ddot{\gamma}_{3}(s), \epsilon \ddot{\gamma}_{2}(s)\right),
\end{aligned}
$$

where $\epsilon=\operatorname{sgn}\left(\left(\ddot{\gamma}_{2}(s)\right)^{2}-\left(\ddot{\gamma}_{3}(s)\right)^{2}\right)$. In this sense, the Frenet formulas for the curve $\gamma$ are $[17,18]$

$$
\begin{aligned}
& \dot{\mathbf{T}}=\kappa \mathbf{N}, \\
& \dot{\mathbf{N}}=\tau \mathbf{B}, \\
& \dot{\mathbf{B}}=\tau \mathbf{N} .
\end{aligned}
$$

Let $M^{2}$ be a surface in the pseudo-Galilean space $\mathbb{G}_{3}^{1}$ parameterized by

$$
\mathbf{r}\left(u_{1}, u_{2}\right)=\left(r_{1}\left(u_{1}, u_{2}\right), r_{2}\left(u_{1}, u_{2}\right), r_{3}\left(u_{1}, u_{2}\right)\right)
$$

Denote $\left(r_{k}\right)_{u_{i}}=\partial r_{k} / \partial u_{i}$ and $\left(r_{k}\right)_{u_{i} u_{j}}=\partial^{2} r_{k} / \partial u_{i} \partial u_{j}, 1 \leq k \leq 3$ and $1 \leq i, j \leq 2$. Then such a surface is admissible if and only if $\left(r_{1}\right)_{u_{i}} \neq 0$ for some $i=1,2$. The coefficients of the first fundamental form of $M^{2}$ are

$$
\begin{aligned}
& g_{i}=\left(r_{1}\right)_{u_{i}}, \\
& h_{i j}=\left(0,\left(r_{2}\right)_{u_{i}},\left(r_{3}\right)_{u_{i}}\right) \cdot\left(0,\left(r_{2}\right)_{u_{j}},\left(r_{3}\right)_{u_{j}}\right), \\
& i, j=1,2,
\end{aligned}
$$

or, in matrix form,

$$
d s^{2}=\left(\begin{array}{cc}
d s_{1}^{2} & 0 \\
0 & d s_{2}^{2}
\end{array}\right)
$$

where $d s_{1}^{2}=\left(g_{1} d u_{1}+g_{2} d u_{2}\right)^{2}$ and $d s_{2}^{2}=h_{11} d u_{1}^{2}+$ $2 h_{12} d u_{1} d u_{2}+h_{22} d u_{2}^{2}$. 
Let us define the function $W$ as

$$
W=\sqrt{\left|\left(\left(r_{1}\right)_{u_{1}}\left(r_{2}\right)_{u_{2}}-\left(r_{1}\right)_{u_{2}}\left(r_{2}\right)_{u_{1}}\right)^{2}-\left(\left(r_{1}\right)_{u_{1}}\left(r_{3}\right)_{u_{2}}-\left(r_{1}\right)_{u_{2}}\left(r_{3}\right)_{u_{1}}\right)^{2}\right|} .
$$

Thus a side tangential vector is defined by

$$
\mathbf{S}=\frac{1}{W}\left[\left(r_{1}\right)_{u_{1}} \mathbf{r}_{u_{2}}-\left(r_{1}\right)_{u_{2}} \mathbf{r}_{u_{1}}\right],
$$

which can be a spacelike isotropic or a timelike isotropic vector. The unit normal vector field $\mathbf{U}$ of $M^{2}$ is given by

$$
\begin{aligned}
\mathbf{U} & =\frac{1}{W}\left(0,\left(r_{1}\right)_{u_{1}}\left(r_{3}\right)_{u_{2}}-\left(r_{1}\right)_{u_{2}}\left(r_{3}\right)_{u_{1}},\left(r_{1}\right)_{u_{1}}\left(r_{2}\right)_{u_{2}}\right. \\
& \left.-\left(r_{1}\right)_{u_{2}}\left(r_{2}\right)_{u_{1}}\right) .
\end{aligned}
$$

The second fundamental form II of $M^{2}$ and its coefficients are defined by

$$
\begin{aligned}
\mathbf{I I} & =L_{11} d u_{1}^{2}+2 L_{12} d u_{1} d u_{2}+L_{22} d u_{2}^{2}, \\
L_{i j} & =\epsilon \frac{1}{g_{1}}\left(g_{1}\left(0,\left(r_{2}\right)_{u_{i} u_{j}},\left(r_{3}\right)_{u_{i} u_{j}}\right)\right. \\
& \left.-\left(g_{i}\right)_{u_{j}}\left(0,\left(r_{2}\right)_{u_{1}},\left(r_{3}\right)_{u_{1}}\right)\right) \cdot \mathbf{U}=\epsilon \\
& \cdot \frac{1}{g_{2}}\left(g_{2}\left(0,\left(r_{2}\right)_{u_{i} u_{j}},\left(r_{3}\right)_{u_{i} u_{j}}\right)\right. \\
& \left.-\left(g_{i}\right)_{u_{j}}\left(0,\left(r_{2}\right)_{u_{2}},\left(r_{3}\right)_{u_{2}}\right)\right) \cdot \mathbf{U},
\end{aligned}
$$

where $\epsilon=\mathbf{U} \cdot \mathbf{U}=-\mathbf{S} \cdot \mathbf{S}= \pm 1$. Two types of admissible surfaces can be distinguished: spacelike surfaces having timelike unit normals $(\epsilon=-1)$ and timelike ones having spacelike unit normals $(\epsilon=1)$. The third fundamental form of $M^{2}$ is

$$
\text { III }=P_{11} d u_{1}^{2}+2 P_{12} d u_{1} d u_{2}+P_{22} d u_{2}^{2},
$$

where

$$
\begin{aligned}
P_{11} & =-\epsilon \frac{g_{2}^{2} L_{11}^{2}-2 g_{1} g_{2} L_{11} L_{12}+g_{1}^{2} L_{12}^{2}}{W^{2}}, \\
P_{12} & \\
& =-\epsilon \frac{g_{2}^{2} L_{11} L_{12}-g_{1} g_{2}\left(L_{11} L_{22}+L_{12}^{2}\right)+g_{1}^{2} L_{12} L_{22}}{W^{2}}, \\
P_{22} & =-\epsilon \frac{g_{2}^{2} L_{12}^{2}-2 g_{1} g_{2} L_{11} L_{22}+g_{1}^{2} L_{22}^{2}}{W^{2}} .
\end{aligned}
$$

The Gaussian curvature and the mean curvature of $M^{2}$ are, respectively, defined by

$$
\begin{aligned}
& K=-\epsilon \frac{L_{11} L_{22}-L_{12}^{2}}{W^{2}}, \\
& H=-\epsilon \frac{g_{2}^{2} L_{11}-2 g_{1} g_{2} L_{12}+g_{1}^{2} L_{22}}{2 W^{2}} .
\end{aligned}
$$

A surface in $\mathbb{G}_{3}^{1}$ is said to be minimal if its mean curvature vanishes.

\section{Hasimoto Surfaces with Null Curvature in $\mathbb{G}_{3}^{1}$}

Let $I_{s}$ and $I_{t}$ be the open intervals of $\mathbb{R}$ and $D=I_{s} \times I_{t}$ open domain of $\mathbb{R}^{2}$.

A Hasimoto surface $M^{2}$ is the surface traced out by a curve in $\mathbb{G}_{3}^{1}$ as it evolves over time according to this evolution equation:

$$
\mathbf{r}_{t}=\mathbf{r}_{s} \times \mathbf{r}_{s s}
$$

where $\mathbf{r}: D \subset \mathbb{R}^{2} \rightarrow \mathbb{G}_{3}^{1}$ is smooth and regular mapping such that $M^{2}=\mathbf{r}(D)$.

In order to parameterize by arc length the curve $\mathbf{r}(s, t)$ for $\forall t \in I_{t}$, the parameterization of the surface in $\mathbb{G}_{3}^{1}$ may be chosen as follows:

$$
\mathbf{r}(s, t)=\left(s+u(t), r_{2}(s, t), r_{3}(s, t)\right),
$$

where $u=u(t)$ is a smooth function of one variable on $I_{t}$. After using the pseudo-Galilean cross product given by (5) and (21), it can be easily seen that the function $u(t)$ is a constant function. In this sense, we get the parameterization of a Hasimoto surface $M^{2}$ in $\mathbb{G}_{3}^{1}$ as

$$
\mathbf{r}(s, t)=\left(s+\lambda, r_{2}(s, t), r_{3}(s, t)\right)
$$

for arbitrary constant $\lambda$. Note that the Hasimoto surface given by (23) is always admissible; that is, it has no pseudoEuclidean tangent planes, if $\left(r_{2}\right)_{t} \neq 0$.

Now, let $M^{2}$ be a Hasimoto surface given by (23) in $\mathbb{G}_{3}^{1}$. Denote the associated Frenet frame field of the curve $r=$ $r(s, t)$ for $\forall t \in I_{t}$ by $\{\mathbf{T}, \mathbf{N}, \mathbf{B}\}$. Then, the derivative of $\mathbf{T}$ with respect to $t$ has the expression

$$
\mathbf{T}_{t}=\varphi_{1} \mathbf{N}+\varphi_{2} \mathbf{B}
$$

for some smooth functions $\varphi_{1}, \varphi_{2}$ on $D$. By (21) and (24), we get

$$
\begin{aligned}
& \varphi_{1}=\varepsilon \kappa \tau, \\
& \varphi_{2}=\varepsilon \kappa_{s} .
\end{aligned}
$$

Moreover, applying the compatibility condition $\mathbf{T}_{s t}=\mathbf{T}_{t s}$ yields that

$$
\kappa_{t}=\varepsilon\left((\kappa \tau)_{s}+\kappa_{s} \tau\right) .
$$

In similar ways, we deduce

$$
\begin{aligned}
& \mathbf{N}_{t}=\varepsilon\left(\frac{\kappa_{s s}}{\kappa}+\tau^{2}\right) \mathbf{B}, \\
& \mathbf{B}_{t}=\varepsilon\left(\frac{\kappa_{s s}}{\kappa}+\tau^{2}\right) \mathbf{N} .
\end{aligned}
$$


The compatibility conditions $\mathbf{N}_{s t}=\mathbf{N}_{t s}$ and $\mathbf{B}_{s t}=\mathbf{B}_{t s}$ give

$$
\tau_{t}=\varepsilon\left(\frac{\kappa_{s s}}{\kappa}\right)_{s}+2 \varepsilon \tau \tau_{s s}
$$

Summing up, we have the following result.

Lemma 1. Let $M^{2}$ be a Hasimoto surface given by (23) in $\mathbb{G}_{3}^{1}$. Then the following equations hold:

(i) $\kappa_{t}=\varepsilon\left((\kappa \tau)_{s}+\kappa_{s} \tau\right)$ and $\tau_{t}=\varepsilon\left(\kappa_{s s} / \kappa\right)_{s}+2 \varepsilon \tau \tau_{s s}$;

(ii)

$$
\left[\begin{array}{c}
\mathbf{T}_{t} \\
\mathbf{N}_{t} \\
\mathbf{B}_{t}
\end{array}\right]=\left[\begin{array}{ccc}
0 & \varepsilon \kappa \tau & \varepsilon \kappa_{s} \\
0 & 0 & \varepsilon\left(\frac{\kappa_{s s}}{\kappa}+\tau^{2}\right) \\
0 & \varepsilon\left(\frac{\kappa_{s s}}{\kappa}+\tau^{2}\right) & 0
\end{array}\right]\left[\begin{array}{c}
\mathbf{T} \\
\mathbf{N} \\
\mathbf{B}
\end{array}\right],
$$

where $\{\mathbf{T}, \mathbf{N}, \mathbf{B}\}$ is the Frenet frame field of the curve $r=r(s, t)$ for $\forall t \in I_{t}$.

Example 2. Let us consider the spacelike admissible Hasimoto surface $M^{2}$ in $\mathbb{G}_{3}^{1}$ parameterized by

$$
\mathbf{r}(s, t)=\left(s-3, t s-2, \frac{s^{3}}{6}\right), \quad-2.5 \leq s, t \leq 2.5 .
$$

It can be easily seen that

$$
\begin{aligned}
& \mathbf{T}(s, t)=\left(1, t, \frac{s^{2}}{2}\right), \\
& \mathbf{N}(s, t)=(0,0,1), \\
& \mathbf{B}(s, t)=(0,-1,0), \\
& \mathcal{\kappa}(s, t)=s, \\
& \tau(s, t)=0 .
\end{aligned}
$$

We plot the surface $M^{2}$ as in Figure 1.

Example 3. Take the timelike admissible Hasimoto surface $M^{2}$ in $\mathbb{G}_{3}^{1}$ as

$$
\mathbf{r}(s, t)=(s, \cosh (s+t)-1, \sinh (s+t)),
$$

$$
-1.3 \leq s, t \leq 1.3 \text {. }
$$

It follows that

$$
\begin{aligned}
& \mathbf{T}(s, t)=(1, \sinh (s+t), \cosh (s+t)), \\
& \mathbf{N}(s, t)=(0, \cosh (s+t), \sinh (s+t)), \\
& \mathbf{B}(s, t)=(0, \sinh (s+t), \cosh (s+t)), \\
& \kappa(s, t)=1, \\
& \tau(s, t)=1 .
\end{aligned}
$$

We plot the surface $M^{2}$ as in Figure 2 .

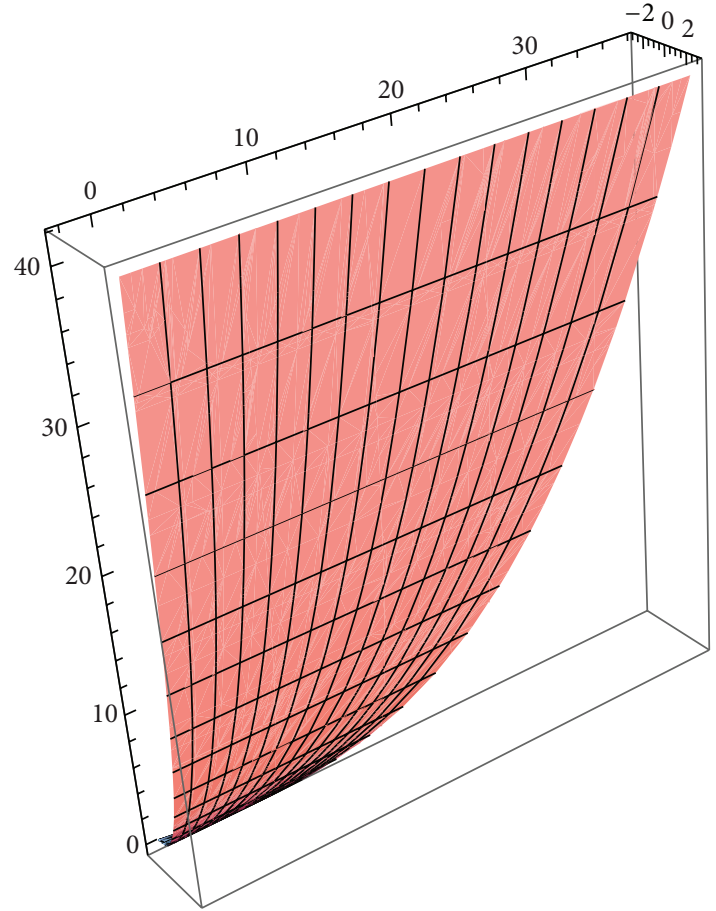

Figure 1

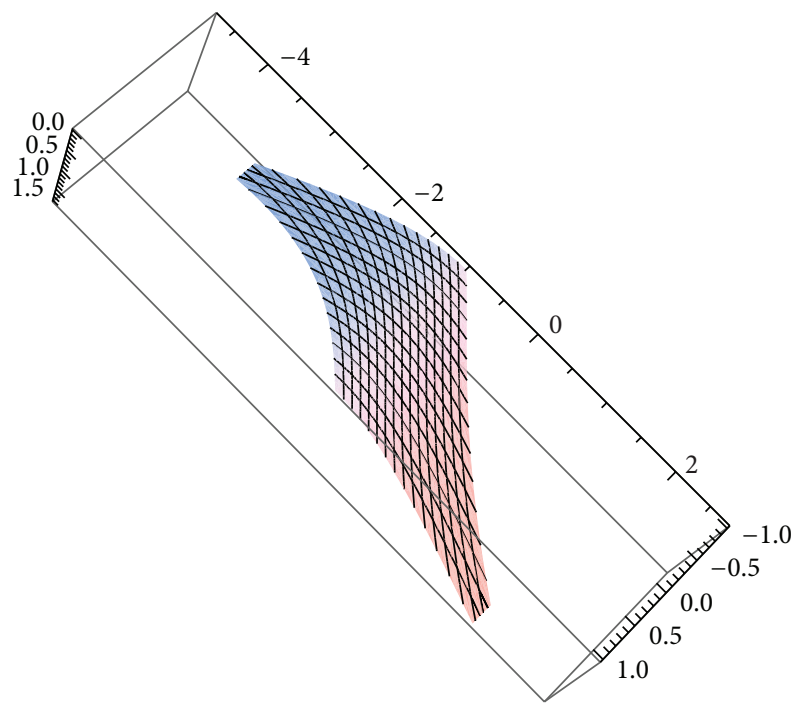

FIGURE 2

Theorem 4. Let $M^{2}$ be a Hasimoto surface given by (23) in $\mathbb{G}_{3}^{1}$. Then

(i) $M^{2}$ has null Gaussian curvature in $\mathbb{G}_{3}^{1}$ if and only if $\varepsilon\left(\kappa_{s s} / \kappa\right)+(\varepsilon-1) \tau^{2}=0 ;$

(ii) $M^{2}$ is a minimal Hasimoto surface in $\mathbb{G}_{3}^{1}$ if and only if $\kappa_{s s}+\kappa \tau^{2}=0$, where $\kappa$ and $\tau$ are, respectively, the curvature and the torsion of the curve $r=r(s, t)$ for $\forall t \in I_{t}$. 
Proof. Let $M^{2}$ be a Hasimoto surface given by (23) in $\mathbb{G}_{3}^{1}$. Then the unit normal vector field $\mathbf{U}$ of $M^{2}$ is

$$
\mathbf{U}(s, t)=\frac{\left(0,\left(r_{2}\right)_{s s},\left(r_{3}\right)_{s s}\right)}{\sqrt{\left|\left[\left(r_{2}\right)_{s s}\right]^{2}-\left[\left(r_{3}\right)_{s s}\right]^{2}\right|}}=\mathbf{N}(s, t),
$$

where $\mathbf{N}(s, t)$ is the principal normal vector field of the curve $\mathbf{r}=\mathbf{r}(s, t)$ for $\forall t \in I_{t}$. Thus, we have

$$
\begin{aligned}
g_{1} & =1, \\
g_{2} & =0, \\
L_{11} & =\varepsilon \kappa, \\
L_{12} & =\varepsilon \kappa \tau, \\
L_{22} & =\kappa_{s s}+\kappa \tau^{2} .
\end{aligned}
$$

From (19) and (35), we deduce that

$$
K=\frac{\varepsilon \kappa \kappa_{s s}+(\varepsilon-1) \kappa^{2} \tau^{2}}{\kappa^{2}}
$$

which gives the first statement.

Next by substituting (35) into (20), we obtain that $M^{2}$ is a minimal Hasimoto surface if and only if

$$
\kappa_{s s}+\kappa \tau^{2}=0
$$

Thus the proof is completed.

Gheorghe Tzitzeica (1873-1939) introduced a class of curves, nowadays called Tzitzeica curves, and a class of surfaces of the 3-dimensional Euclidean space, called Tzitzeica surface.

A Tzitzeica surface is a spatial surface for which the ratio of its Gaussian curvature $K$ and the distance $d$ from the origin to the tangent plane at any arbitrary point of the surface satisfy $K=\lambda d^{4}$ for a constant $\lambda$. This class of surface is of great interest, having important applications both in mathematics and in physics (see [15]). The relation between Tzitzeica curves and surfaces is the following: For a Tzitzeica surface with negative Gaussian curvature, the asymptotic lines are Tzitzeica curves [19-21].

It is easy to prove that the tangent plane at an arbitrary point of any Hasimoto surface passes through the origin of $\mathbb{G}_{3}^{1}$. That is why all Hasimoto surfaces having null Gaussian curvature satisfy Tzitzeica condition in $\mathbb{G}_{3}^{1}$.

Therefore the following result can be given without proof.

Theorem 5. Let $M^{2}$ be a Hasimoto surface given by (23) in $\mathbb{G}_{3}^{1}$. Then it is a Tzitzeica surface if and only if $\varepsilon\left(\kappa_{s s} / \kappa\right)+(\varepsilon-1) \tau^{2}=$ 0 , where $\kappa$ and $\tau$ are, respectively, the curvature and the torsion of the curver $=r(s, t)$ for $\forall t \in I_{t}$.

On the other hand, the third fundamental form III of a surface in $\mathbb{G}_{3}^{1}$ may be introduced in the analogous way as in Euclidean space. Let $M^{2}$ be a surface and $\mathbf{U}$ its unit normal vector in $\mathbb{G}_{3}^{1}$. If $M^{2}$ is spacelike (timelike) in $\mathbb{G}_{3}^{1}$, then the end points of associated position vectors of $\mathbf{U}$ lie on a unit spacelike sphere $z^{2}-y^{2}=1$ (unit timelike sphere $y^{2}-z^{2}=1$ ). The mapping obtained in such a way is called the Gauss mapping or the spherical mapping in $\mathbb{G}_{3}^{1}$. The set of all end points of $\mathbf{U}$ is called the spherical image of $M^{2}$ in $\mathbb{G}_{3}^{1}$. In this sense, the third fundamental form is indeed the first fundamental form of the spherical image (cf. [13]). Thus the following result for the Hasimoto surfaces in $\mathbb{G}_{3}^{1}$ can be given.

Corollary 6. Let $M^{2}$ be a Hasimoto surface given by (23) in $\mathbb{G}_{3}^{1}$. Then its third fundamental form is singular; that is, $\operatorname{det} I I I=0$.

Proof. Let us consider the Hasimoto surface $M^{2}$ given by (23) in $\mathbb{G}_{3}^{1}$. Then from (35) we have $g_{2}=0$ as one of the coefficients of the first fundamental form of $M^{2}$. This immediately implies from (18) that $P_{11} P_{22}-P_{12}^{2}=0$, which completes the proof.

\section{Curves on Hasimoto Surfaces in $\mathbb{G}_{3}^{1}$}

There exists a frame field, also called the Darboux frame field, for the curves lying on surfaces apart from the Frenet frame field. For details, see $[22,23]$. Let $\gamma$ be a curve lying on the surface $M^{2}$ with unit normal vector field $\mathbf{U}$. By taking $\mathbf{T}=$ $\gamma_{*}(d / d t)$ one can get a new frame field $\{\mathbf{T}, \mathbf{T} \times \mathbf{U}, \mathbf{U}\}$ which is the Darboux frame field of $\gamma$ with respect to $M^{2}$.

On the other hand, the second derivative $\ddot{\gamma}$ of the curve $\gamma$ on $M^{2}$ has a component perpendicular to $M^{2}$ and a component tangent to $M^{2}$; that is,

$$
\ddot{\gamma}=\ddot{\gamma}^{\top}+\ddot{\gamma}^{\perp}
$$

where the dot "." denotes the derivative with respect to the parameter of the curve. The norms $\left\|\ddot{\gamma}^{\top}\right\|$ and $\left\|\ddot{\gamma}^{\perp}\right\|$ are called the geodesic curvature and the normal curvature of $\gamma$ on $\mathrm{M}^{2}$, respectively. The curve $\gamma$ is called geodesic (resp., asymptotic line) if and only if its geodesic curvature $\kappa_{g}$ (resp., normal curvature $\kappa_{n}$ ) vanishes.

In our framework, the following results provide some characterizations for the parameter curves of the Hasimoto surfaces to be geodesics and asymptotic lines in $\mathbb{G}_{3}^{1}$.

Theorem 7. Let $M^{2}$ be a Hasimoto surface given by (23) in $\mathbb{G}_{3}^{1}$. Then

(i) the s-parameter curves on $M^{2}$ are geodesics of $M^{2}$,

(ii) the t-parameter curves on $M^{2}$ are geodesics of $M^{2}$ if and only if $(\kappa \tau)_{s}+\kappa_{s} \tau$ vanishes, where $\kappa$ and $\tau$ are, respectively, the curvature and the torsion of the curve $r(s, t)$ for $\forall t \in I_{t}$.

Proof. Let us assume that $M^{2}$ is a Hasimoto surface given by (23) in $\mathbb{G}_{3}^{1}$. Then the geodesic curvature of the $s$-parameter curves on $M^{2}$ is the tangential component of $\mathbf{r}_{s s}$; that is,

$$
\kappa_{g}=\mathbf{S} \cdot \mathbf{r}_{s s}=\frac{1}{\sqrt{\left|\left[\left(r_{2}\right)_{s s}\right]^{2}-\left[\left(r_{3}\right)_{s s}\right]^{2}\right|}}\left(\mathbf{r}_{t} \cdot \mathbf{r}_{s s}\right) .
$$

From (21), we derive that the geodesic curvature $\kappa_{g}$ of the $s$ parameter curves vanishes. This gives first statement. 
Similarly, the geodesic curvature of the $t$-parameter curves on $M^{2}$ is given by

$$
\kappa_{g}=\mathbf{S} \cdot \mathbf{r}_{t t}=\frac{1}{\sqrt{\left|\left[\left(r_{2}\right)_{s s}\right]^{2}-\left[\left(r_{3}\right)_{s s}\right]^{2}\right|}}\left(\mathbf{r}_{t} \cdot \mathbf{r}_{t t}\right)
$$

By considering Lemma 1 into (40), we deduce that $\kappa_{g}$ is identically zero if and only if $(\kappa \tau)_{s}+\kappa_{s} \tau$ vanishes, which implies statement (ii).

Theorem 8. Let $M^{2}$ be a Hasimoto surface given by (23) in $\mathbb{G}_{3}^{1}$. Then

(i) the s-parameter curves on $M^{2}$ cannot be asymptotic lines of $M^{2}$,

(ii) the t-parameter curves on $M^{2}$ are asymptotic lines of $M^{2}$ if and only if $\kappa_{s s}+\kappa \tau^{2}$ vanishes, where $\kappa$ and $\tau$ are, respectively, the curvature and the torsion of the curve $r(s, t)$ for $\forall t \in I_{t}$.

Proof. Suppose that $M^{2}$ is a Hasimoto surface given by (23) in $\mathbb{G}_{3}^{1}$. Then the normal curvature of the $s$-parameter curves on $M^{2}$ is the normal component of $\mathbf{r}_{s s}$; that is,

$$
\kappa_{n}=\mathbf{U} \cdot \mathbf{r}_{s s}=\mathbf{N} \cdot(\kappa \mathbf{N})=\kappa .
$$

This means that the $s$-parameter curves are asymptotic lines if and only if $\kappa=0$, which is not possible since the fact that $r=r(s, t)$ is a regular mapping. This gives the first statement.

The normal curvature of the $t$-parameter curves on $M^{2}$ is defined by

$$
\kappa_{n}=\mathbf{U} \cdot \mathbf{r}_{t t}=\kappa_{s s}+\kappa \tau^{2} .
$$

Equation (42) yields that the $t$-parameter curves are asymptotic lines if and only if $\left(\kappa_{s s} / \kappa\right)+\tau^{2}=0$, which completes the proof.

Comparing Theorems 4 and 8 , we have the following.

Corollary 9. A Hasimoto surface given by (23) in $\mathbb{G}_{3}^{1}$ is minimal if and only if its $t$-parameter curves are asymptotic lines.

A curve $\gamma$ on a regular surface $M^{2}$ is called a principal curve if and only if its velocity vector field always points in a principal direction. Moreover, a surface $M^{2}$ is called a principal surface if and only if its parameter curves are principal curves.

A principal curve $\gamma$ on a surface in $\mathbb{G}_{3}^{1}$ is determined by the following formula:

$$
\operatorname{det}(\dot{\gamma}, \mathbf{U}, \dot{\mathbf{U}})=0,
$$

where $\mathbf{U}$ is the unit normal vector field of the surface. Thus, we have the following results.
Theorem 10. Let $M^{2}$ be a Hasimoto surface given by (23) in $\mathbb{G}_{3}^{1}$. Then

(i) the s-parameter curves on $M^{2}$ are principal curves of $M^{2}$ if and only if $\tau$ vanishes, where $\tau$ is the torsion of the curve $\mathbf{r}(s, t)$ for all $t$;

(ii) the t-parameter curves on $M^{2}$ are principal curves of $M^{2}$.

Proof. Assume that $M^{2}$ is a Hasimoto surface given by (23) in $\mathbb{G}_{3}^{1}$. Then the $s$-parameter curves are the principal curves if and only if

$$
\operatorname{det}\left(r_{s}, \mathbf{U}, \mathbf{U}_{s}\right)=\operatorname{det}\left(r_{s}, \mathbf{N}, \mathbf{N}_{s}\right)=\tau \operatorname{det}(\mathbf{T}, \mathbf{N}, \mathbf{B}),
$$

where $\tau$ is the torsion and $\{\mathbf{T}, \mathbf{N}, \mathbf{B}\}$ is the Frenet frame field of the curve $\mathbf{r}=\mathbf{r}(s, t)$ for all $t$ such that $\operatorname{det}(\mathbf{T}, \mathbf{N}, \mathbf{B})=1$. By (43), it follows that the $s$-parameter curves are principal if and only if their torsions vanish, which gives the first statement.

In a similar way, by using (24), it is easily seen that $\operatorname{det}\left(\mathbf{r}_{t}, \mathbf{U}, \mathbf{U}_{t}\right)=0$, which specify that $t$-parameter curves are the principal curves.

Therefore the proof is completed.

As a consequence of Theorem 10, the following result can be given.

Corollary 11. A Hasimoto surface $M^{2}$ given by (23) in $\mathbb{G}_{3}^{1}$ is a principal surface if and only if the torsions of s-parameter curves vanish.

\section{Conflict of Interests}

The authors declare that there is no conflict of interests regarding the publication of this paper.

\section{Acknowledgments}

The second author's work was supported by the DAAD Fellowship for University Professors and Researchers A/14/02337 at Technical University Berlin, December 2014 to January 2015. She thanks Professor Udo Simon for being her host Professor and for his kind hospitality. The drawings in the present paper are made with Wolfram Mathematica 7.0.

\section{References}

[1] M. Barros, J. L. Cabrerizo, M. Fernández, and A. Romero, "Magnetic vortex filament flows," Journal of Mathematical Physics, vol. 48, no. 8, Article ID 082904, pp. 1-27, 2007.

[2] M. Barros and A. Romero, "Magnetic vortices," Europhysics Letters, vol. 77, no. 3, Article ID 34002, 5 pages, 2007.

[3] H. Hasimoto, "A soliton on a vortex filament," Journal of Fluid Mechanics, vol. 51, no. 03, p. 477, 1972.

[4] T. A. Ivey, "Helices, Hasimoto surfaces and Bäcklund transformations," Canadian Mathematical Bulletin, vol. 43, no. 4, pp. 427-439, 2000.

[5] C. Rogers and W. K. Schief, "Intrinsic geometry of the NLS equation and its auto-backlund transformation," Studies in Applied Mathematics, vol. 101, no. 3, pp. 267-287, 1998. 
[6] N. H. Abdel-All, R. A. Hussien, and T. Youssef, "Hasimoto surfaces," Life Science Journal, vol. 9, no. 3, pp. 556-560, 2012.

[7] M. Erdogdu and M. Ozdemir, "Geometry of Hasimoto surfaces in Minkowski 3-space," Mathematical Physics, Analysis and Geometry, vol. 17, no. 1-2, pp. 169-181, 2014.

[8] B. Divjak and Ž. M. Šipuš, "Some special surfaces in the pseudoGalilean space," Acta Mathematica Hungarica, vol. 118, no. 3, pp. 209-226, 2008.

[9] B. Divjak and Ž. M. Šipuš, "Minding isometries of ruled surfaces in pseudo-Galilean space," Journal of Geometry, vol. 77, no. 1-2, pp. 35-47, 2003.

[10] B. Divjak and Ž. M. Šipuš, "Special curves on ruled surfaces in Galilean and pseudo-Galilean spaces," Acta Mathematica Hungarica, vol. 98, pp. 175-187, 2003.

[11] Ž. M. Šipuš, "Ruled Weingarten surfaces in the Galilean space," Periodica Mathematica Hungarica, vol. 56, pp. 213-225, 2008.

[12] Ž. M. Šipuš and B. Divjak, "Translation surfaces in the Galilean space," Glasnik Matematicki, Serija III, vol. 46, no. 2, pp. 455469, 2011.

[13] Ž. M. Šipuš and B. Divjak, "Surfaces of constant curvature in the pseudo-Galilean space," International Journal of Mathematics and Mathematical Sciences, vol. 2012, Article ID 375264, 28 pages, 2012.

[14] M. E. Aydin and A. Mihai, "Classification of quasi-sum production functions with Allen determinants," Filomat, vol. 29, no. 6, pp. 1351-1359, 2015.

[15] G. E. Vilcu, "A geometric perspective on the generalized CobbDouglas production functions," Applied Mathematics Letters, vol. 24, no. 5, pp. 777-783, 2011.

[16] D. W. Yoon, "Surfaces of revolution in the three dimensional pseudo-Galilean space," Glasnik Matematicki Series III, vol. 48, no. 2, pp. 415-428, 2013.

[17] Z. Erjavec, "On generalization of helices in the Galilean and the pseudo-Galilean space," Journal of Mathematics Research, vol. 6, no. 3, pp. 39-50, 2014.

[18] M. K. Karacan and Y. Tuncer, "Tubular surfaces of Weingarten types in GALilean and pseudo-GALilean," Bulletin of Mathematical Analysis and Applications, vol. 5, no. 2, pp. 87-100, 2013.

[19] M. E. Aydin and A. Mihai, "Translation hypersurfaces and Tzitzeica translation hypersurfaces of the Euclidean space," Proceedings of the Romanian Academy Series A. To appear.

[20] O. Constantinescu and M. Crasmareanu, "A new Tzitzeica hypersurface and cubic Finslerian metrics of Berwald type," Balkan Journal of Geometry and Its Applications, vol. 16, no. 2, pp. 27-34, 2011.

[21] M. Crasmareanu, "Cylindrical Tzitzeica curves implies forced harmonic oscillators," Balkan Journal of Geometry and Its Applications, vol. 7, no. 1, pp. 37-42, 2002.

[22] M. P. do Carmo, Differential Geometry of Curves and Surfaces, Prentice-Hall, Englewood Cliffs, NJ, USA, 1976.

[23] A. Gray, Modern Differential Geometry of Curves and Surfaces with Mathematica, CRC Press, Boca Raton, Fla, USA, 2nd edition, 1998. 


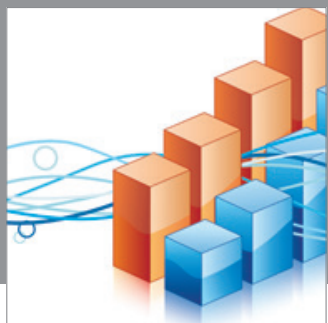

Advances in

Operations Research

mansans

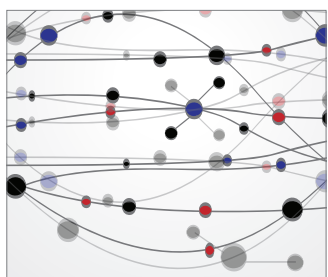

The Scientific World Journal
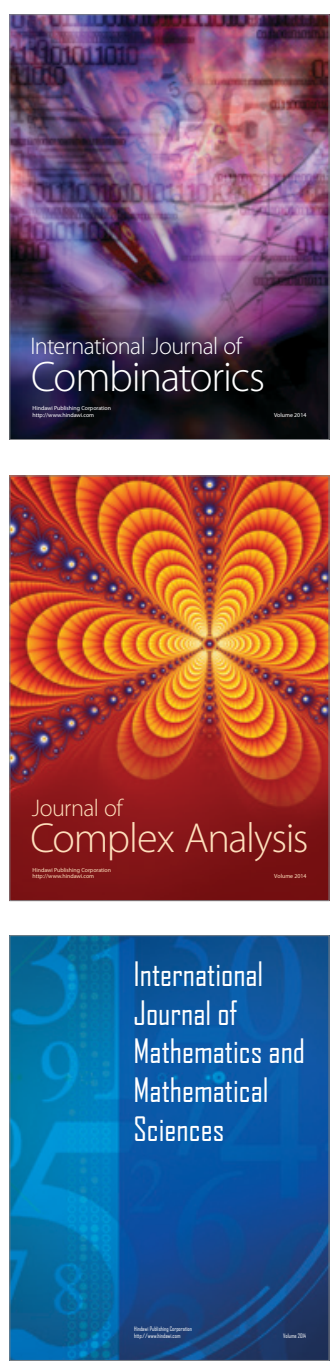
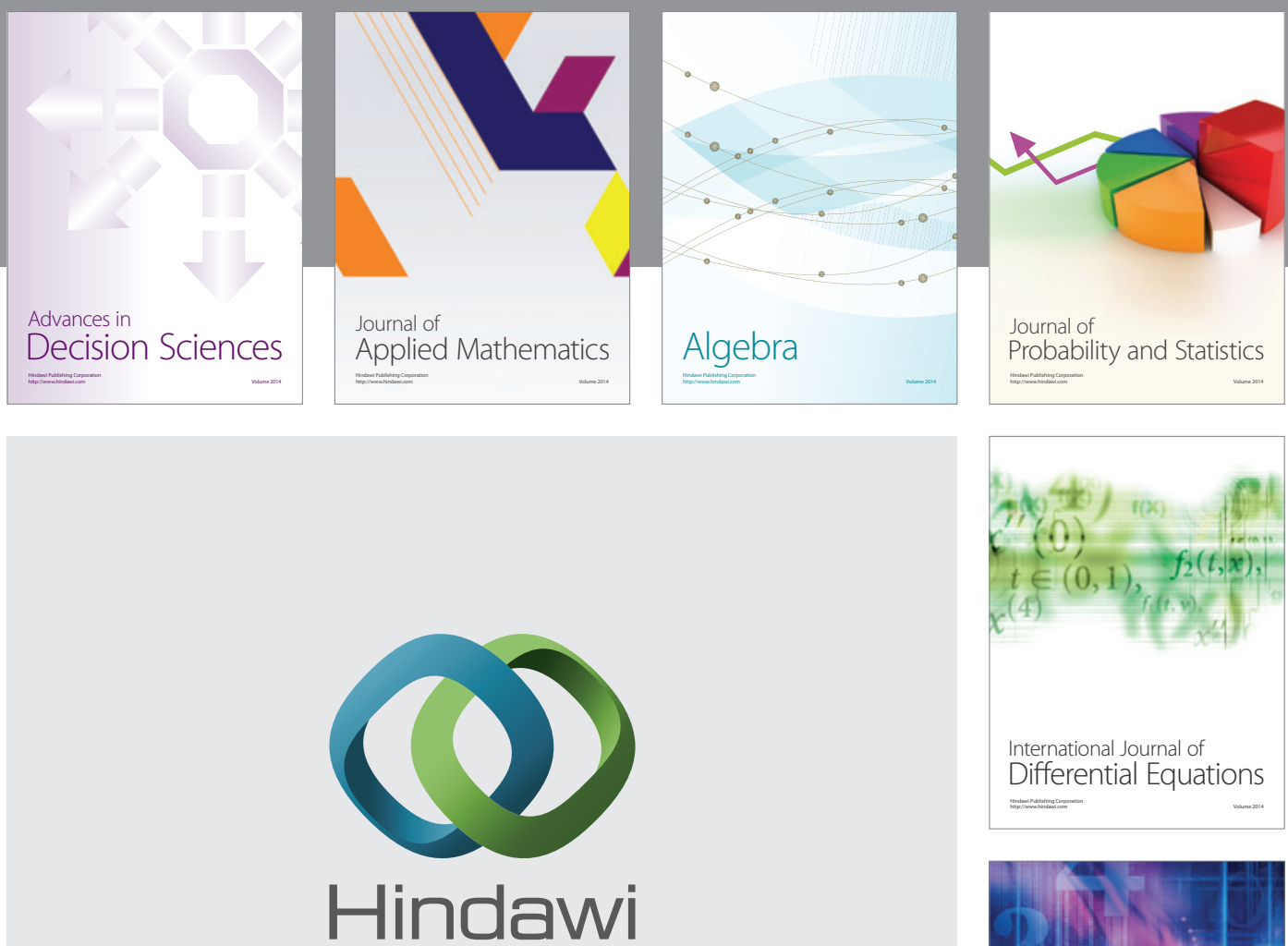

Submit your manuscripts at http://www.hindawi.com
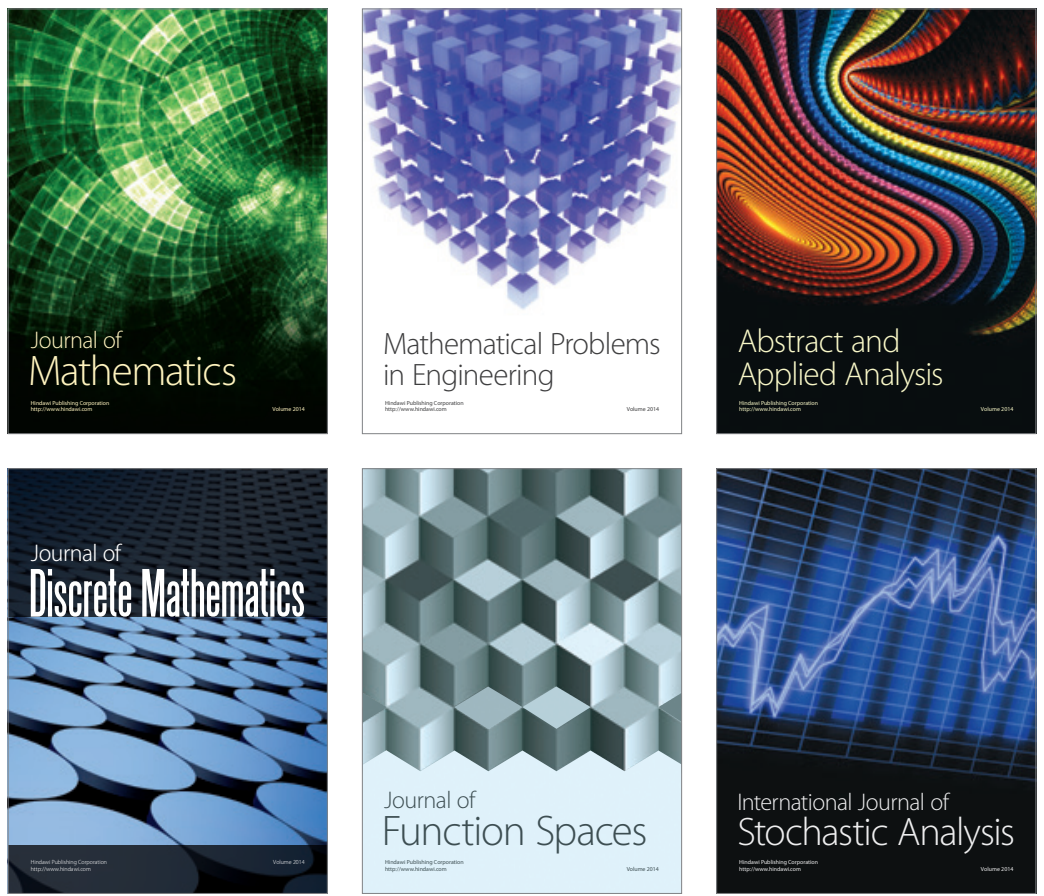

Journal of

Function Spaces

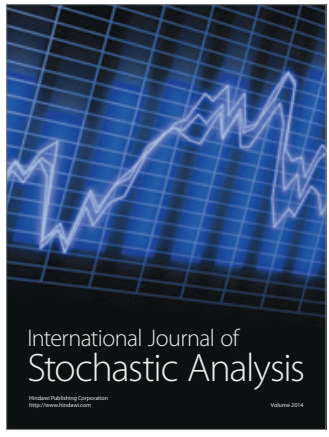

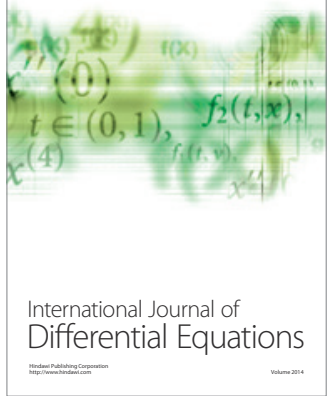
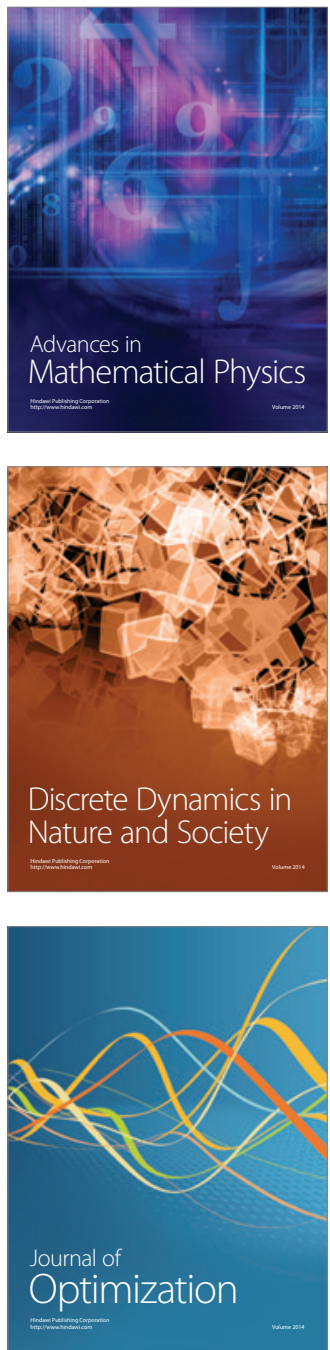